\title{
Effects of acoustic heterogeneity on the breast thermoacoustic tomography
}

Yuan Xu, Lihong V. Wang

Yuan Xu, Lihong V. Wang, "Effects of acoustic heterogeneity on the breast thermoacoustic tomography," Proc. SPIE 4960, Biomedical Optoacoustics IV, (1 July 2003); doi: $10.1117 / 12.477784$

SPIE. Event: Biomedical Optics, 2003, San Jose, CA, United States 


\title{
Effects of Acoustic Heterogeneity in Breast Thermoacoustic Tomography Yuan Xu and Lihong V. Wang* \\ Optical Imaging Laboratory, Department of Biomedical Engineering \\ Texas A\&M University, 3120 TAMU, College Station, Texas 77843-3120
}

\author{
* Author to whom all correspondence should be addressed. Tel: 979-847-9040; fax: 979-845-4450; \\ electronic mail: LWang@tamu.edu; URL: http://oilab.tamu.edu.
}

\begin{abstract}
The effects of wavefront distortions induced by acoustic heterogeneities in breast thermoacoustic tomography (TAT) are studied. First, amplitude distortions are shown to be insignificant for different scales of acoustic heterogeneities. Next, the effects of phase distortions (errors in time-of-flight) in our numerical studies are investigated, and the spreads of point sources and boundaries caused by the phase distortions are studied. After that, a demonstration showing that the blurring of images can be compensated for by using the distribution of acoustic velocity in the tissues in the reconstructions is presented. Last, the differences in the effects of acoustic heterogeneity and the generation of speckles in breast TAT and breast ultrasound imaging are discussed.
\end{abstract}

Keywords: thermoacoustic tomography, acoustic heterogeneity, wavefront distortion.

\section{INTRODUCTION}

When an electromagnetic pulse is absorbed by biological tissue, the heating and subsequent expansion causes the emission of acoustic signals. This phenomenon is called the thermoacoustic effect. In thermoacoustic tomography (TAT), the thermoacoustic signals from a tissue sample are collected to map the distribution of the radiation absorption within the sample. Radiation absorption is closely related to the physiological and pathological status of the tissue. For example, the microwave absorption rate of cancerous breast tissue is 2-5 times greater than that of the surrounding normal breast tissue. This difference has been attributed to an increase in the amount of bound water and sodium within malignant cells. ${ }^{1-3}$. TAT combines good imaging resolution with high imaging contrast. There are a variety of reconstruction algorithms for TAT. ${ }^{4-9}$ An important assumption in these reconstruction algorithms is that the tissue is acoustically homogeneous. For many medical imaging applications, including imaging of the female breast, this assumption is an approximation. For example, the speed of sound in the breast can vary from $1400 \mathrm{~m} / \mathrm{s}$ to $1550 \mathrm{~m} / \mathrm{s}$. Errors due to the assumption of a constant acoustic speed, which has never been studied in TAT, can potentially have a pronounced effect on image quality. In breast ultrasound tomography (UT), however, wavefront distortion has been studied extensively ${ }^{10-13}$. Amplitude distortion caused by refraction dominates the phase distortion induced by acoustic speed variation in the breast $\mathrm{UT}^{11}$. Refraction occurs where there is a speed mismatch across a tissue interface. Because of refraction, rays from a single source can reach the same receiver by different paths, as shown in Fig. 1. The interference between these rays causes strong amplitude distortion in breast UT. Different deaberration methods have been proposed to compensate for phase distortion in UT. ${ }^{14,15}$ However, they have so far been inadequate to correct the strong amplitude distortion caused by refraction. ${ }^{16}$

The effects of acoustic heterogeneity on breast TAT are estimated to be weaker than those in breast UT for the following reasons. First, the signals in breast TAT are primarily in a lower frequency range (usually below $1.5 \mathrm{MHz}{ }^{17}$ ) than those in UT. Ultrasound scattering in this frequency range is weak. Secondly, in TAT, the acoustic source is induced by electromagnetic absorption; therefore, only one-way distortion on reception wave propagation occurs. As shown in Fig. 2, an acoustic ray, for example $S B_{l} D$, needs to pass through interface $\Sigma$ only once. In contrast, in pure ultrasound imaging, either in the pulse-echo mode or in the transmission mode, ultrasound distortion includes two parts-distortion during transmission and during reception wave propagation. Therefore, the acoustic wave has to pass through the interface at least twice, as shown in $S B_{2} B_{1} D$ in Fig. 1. Third, if the detection distance from the objects are properly chosen, the effects of amplitude distortion can be minimized in breast TAT, as will be explained in Section 3 . 


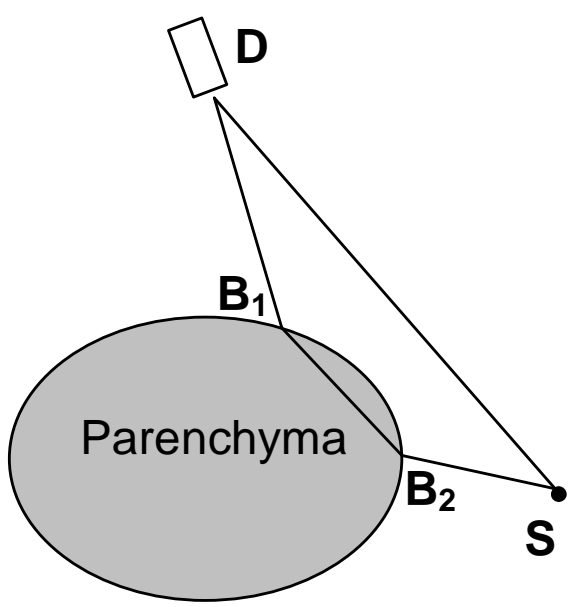

Fig. 1 Illustration of the multipath interference caused by refraction at boundary points $B_{1}$ and $B_{2}$ in breast ultrasound imaging in transmission mode. $S$ is a point source and $D$ is a detector.

In our work, we analyze the effects of amplitude distortion and numerically simulate the effects of phase distortion with the truncated conjugate gradient ${ }^{18}$ (TCG) method. In Section 2, we derive equations for the forward problem in an acoustically homogeneous model, which yields acoustic pressure from a known distribution of microwave absorption. In Section 3, we investigate the effects of refraction on wavefront amplitude and phase in breast TAT. The inversion algorithm of TCG, and the model and parameters used in the numerical simulations, are presented in Section 4. In Section 5, the effects of phase distortion are studied numerically.

\section{THE FORWARD PROBLEM IN A HOMOGENEOUS MODEL}

We begin by deriving a formula for the forward problem for an acoustically homogeneous model, and then modify it, at the end of Section 3, to consider velocity heterogeneity. In the case of thermal confinement, the acoustic wave at point $\mathbf{r}$ and time $t, p_{1}(\mathbf{r}, t)$ can be expressed as follows ${ }^{19}$ :

$$
p_{1}(\mathbf{r}, t)=\frac{v_{s 0} \beta I_{0}}{4 \pi C} \frac{\partial}{\partial t} \iint_{t=t_{f}\left(\mathbf{r}^{\prime}, \mathbf{r}\right)} \frac{\varphi\left(\mathbf{r}^{\prime}\right)}{\left|\mathbf{r}-\mathbf{r}^{\prime}\right|} d \mathbf{r}^{\prime},
$$

where

$$
t_{f}\left(\mathbf{r}^{\prime}, \mathbf{r}\right)=\left|\mathbf{r}-\mathbf{r}^{\prime}\right| / v_{s 0}
$$

is the time-of-flight (TOF) from $\mathbf{r}^{\prime}$ to $\mathbf{r} v_{s 0}$ is the acoustic speed; $C$ is the specific heat; $\beta$ is the coefficient of the volume thermal expansion; $I_{0}$ is a scaling factor proportional to the incident radiation intensity; and $\varphi\left(\mathbf{r}^{\prime}\right)$ describes the to-be-reconstructed microwave absorption properties of the medium at $\mathbf{r}^{\prime}$. The physical meaning of this equation is that, in an acoustically homogenous medium, the pressure $p_{1}$, at a spatial point $\mathbf{r}$ and time $t$, is proportional to the firstorder temporal derivative of the integration of the absorbed microwave energy over a spherical surface [a circle in the two-dimensional (2-D) case]. The spherical surface is centered at $\mathbf{r}$ and has a radius of $t v_{s 0}$. 


\section{THE EFFECT OF ACOUSTIC HETEROGENEITY IN TAT}

A TAT model is shown in Fig. 2. In our imaging system, mineral oil is chosen as the coupling medium for both microwaves and ultrasonic waves. The acoustic speed of light in mineral oil is $1437 \mathrm{~m} / \mathrm{s}^{20}$, which is very close to that in fat. ${ }^{23}$ Therefore, there should be negligible refraction at the boundary between the breast and the mineral oil, and, consequently, we will consider only the effects of acoustical heterogeneity within the breast. More details on our TAT experimental setup can be found in our previous work ${ }^{8}$.

\subsection{Amplitude distortion caused by refraction}

Fig. 1 shows the multipath interference in breast ultrasound imaging in transmission mode. The acoustic ray from source $S$ can travel to detector $D$ by two different paths, $S D$ and $S B_{2} B_{l} D$, due to refraction at the interfaces between different tissues. The interference between the two rays can cause amplitude distortion, ${ }^{11}$ but it is not severe in breast $\mathrm{TAT}^{19}$. Basically, the phenomenon can be explained as follows. For wavelength-scale or smaller heterogeneities, amplitude distortion of the wavefronts is minor due to diffraction when the detectors are placed in the far field of the irregular boundary segment. When the size of the concave segment is larger, or the boundary segment is convex, according to the imaging formula of concave boundaries, only imaginary images exist after the wavefronts from real objects pass through the concave boundary. Equivalently, no two rays from a point source will intersect with each other after passing through the concave boundary segment and no strong amplitude distortion occurs.

\subsection{Phase distortion caused by refraction and speed variation}

If the background is acoustically homogeneous, an acoustic ray from source $S$ in Fig. 2 goes along the straight line $S D$ to reach detector $D$. When there is acoustic heterogeneity, an acoustic ray goes along line $S B_{l} D$ because of refraction at the interface. Assume there is no change in the shape of the acoustic pulse caused by acoustic heterogeneity. The TOF from source $S$ to detector $D$ in the acoustically heterogeneous model is

$$
t_{S B_{1} D}=\int_{S B_{1} D} d l / v_{s}\left(\mathbf{r}^{\prime \prime}\right),
$$

where $v_{s}\left(\mathbf{r}^{\prime \prime}\right)$ is the local acoustic speed, and $\mathbf{r}^{\prime \prime}$ is a point within line $S B_{I} D$. Now, we will show that $t_{S B_{1} D}$ can be approximated to the second order of a small value $\varepsilon=\left(v_{s}\left(\mathbf{r}^{\prime \prime}\right)-v_{s 0}\right) / v_{s 0}$ by $t_{S D}=\int_{S D} d l / v_{s}\left(\mathbf{r}^{\prime \prime}\right)$, where $v_{s 0}$ is the velocity used in the acoustically homogeneous model. According to Fermat's principle, an acoustic ray travels on the fastest path. In another words, $S B_{1} D$ is a local minimum of TOF. Now assume $B_{l}$ is displaced to $B^{\prime}$ by a small distance $q=\left|B B^{\prime}\right|$,

$$
\frac{q}{l_{S D}}=o(\varepsilon)
$$

After expanding $t_{S B^{\prime} D}$ around $t_{S B_{1} D}$ with respect to $q$, we have

$$
t_{S B^{\prime} D}=t_{S B_{1} D}+\left.q \frac{\partial t_{S B^{\prime} D}}{\partial q}\right|_{q=0}+o\left(\varepsilon^{2}\right)
$$

Recalling that $S B_{1} D$ is a local minimum, we have $\left.\frac{\partial t_{S B_{1}^{\prime} D}}{\partial q}\right|_{q=0}=0$. Substituting it into Eq. (5) and assuming $l_{B_{2} B_{1}} / l_{S D}=o(\varepsilon)$, due to the weak acoustic heterogeneity in breast tissue, we have

$$
t_{S D}=\int_{S D} d l / v_{s}\left(\mathbf{r}^{\prime \prime}\right)=t_{S B_{1} D}+o\left(\varepsilon^{2}\right) .
$$


The above result can be understood in the following way. Although the path length of $S B_{1} D$ in Fig. 2 is longer than that of $S D$ and $\left(l_{S B_{1}}+l_{D B_{1}}-l_{S D}\right) / l_{S D}=o(\varepsilon)$, path $S D$ has a longer part within the slow-speed area than path $S B_{1} D$. The combination of the two opposite effects leads to the cancellation of the first-order term of $\varepsilon$ in Eq. (6).

Next we will show that the approximation of $t_{S B_{1} D}$ by $t_{S D}$ includes most of the flight-time variation induced by acoustic heterogeneity. The TOF from source $S$ to detector $D$ in an acoustically homogeneous and heterogeneous model is $l_{S D} / v_{s 0}$ and $t_{S B_{1} D}$, respectively. The difference between them is

$$
\delta t=\left|t_{S B_{1} D}-l_{S D} / v_{s 0}\right|=\left|t_{S B_{1} D}-t_{S D}+t_{S D}-l_{S D} / v_{s 0}\right| \approx\left|o\left(\varepsilon^{2}\right)+t_{S D}-l_{S D} / v_{s 0}\right| \approx o(\varepsilon),
$$

where we used Eq. (6). Combining $\delta t$ with Eq. (6), we have

$$
\frac{\left|t_{S D}-t_{S B_{1} D}\right|}{\delta t}=o(\varepsilon) \text {. }
$$

Therefore, the error in the approximation of $t_{S B_{1} D}$ by $t_{S D}$ is not important. At last, it should be pointed out that our analysis of TOF can be applied to both 2-D and 3-D TAT.

\subsection{Forward formula in an acoustically heterogeneous model}

In our analysis of TOF, we consider only a single interface. The results can be extended to the case involving several interfaces. In general, the TOF from $\mathbf{r}$ to $\mathbf{r}^{\prime}$ can be expressed as

$$
t_{f}\left(\mathbf{r}^{\prime}, \mathbf{r}\right)=\int_{L\left(\mathbf{r}^{\prime}, \mathbf{r}\right)} d l / v_{s}\left(\mathbf{r}^{\prime \prime}\right)+o\left(\varepsilon^{2}\right),
$$

where $L\left(\mathbf{r}^{\prime}, \mathbf{r}\right)$ is the straight line from $\mathbf{r}^{\prime}$ to $\mathbf{r}$, and $\mathbf{r}^{\prime \prime}$ lies within the line $L$. Combining Eq. (9) and Eq. (1), we obtain the forward formula for acoustically heterogeneous TAT.

Our analysis of TOF is in agreement with the results from a more rigid model ${ }^{21}$. It has been reported that the variation in travel time caused directly by acoustic speed heterogeneity is a first-order perturbation and that the effect of ray bending on travel time is a second-order one. For breast tissue, which is weakly acoustically heterogeneous, it is enough to consider the first-order perturbation by computing the integral of the slowness perturbation along straight lines, as shown in Eq. (9).

\section{IMPLEMENTATION AND MODELING OF NUMERICAL SIMULATIONS}

Fig. 3 (a) and (b) illustrate the acoustic and RF absorption models of the breast, respectively. The acoustic model of the breast in our simulations is based on experimental results of the distribution of acoustic speed in the breast ${ }^{22-23}$. The mean velocity in the subcutaneous zone $v_{f}$ and the breast parenchyma $v_{p}$ are set to be $1437 \mathrm{~m} / \mathrm{s}{ }^{23}$ and $1546 \mathrm{~m} / \mathrm{s}$, respectively. A random component, which is a normal distribution with a mean of zero and a variance of $33 \mathrm{~m} / \mathrm{s}$, is added to the velocity distribution to simulate the velocity fluctuations in the subcutaneous zone ${ }^{22}$ and the breast parenchyma. The speed distribution in Fig. 3 was normalized to $1437 \mathrm{~m} / \mathrm{s}$. The RF absorption model of the breast is shown in Fig. 3(b). The RF absorption coefficients in the fat, tumors, and coupling oil are set to be $0.1,3$, and 0 after being normalized to that in the parenchyma. The tumors, shown in 3(b) as dark spots, are placed evenly along the horizontal direction to study the dependence of the distortions in the images based on the tumor locations. We set the radii of the four tumors to about $1.2 \mathrm{~mm}$ to simulate approximately the point-source spread caused by acoustic heterogeneity.

The parameters in our simulations are chosen as follows unless stated otherwise. Noise is added to the generated signals so that the frequency range with a signal-to-noise ratio (SNR) larger than unity is from 0 to $1.5 \mathrm{MHz}$, which approximates our experimental results ${ }^{17}$. The radius of the circle of detection is set to be $125 \mathrm{~mm}$; the angle range of detection is $2 \pi$ with 200 steps. An insufficient number of scanning steps can cause radial aliases in the reconstructed image ${ }^{9}$. Thermoacoustic signals are sampled for $108 \mu$ s at a sampling rate of about $7 \mathrm{MHz}$, which is sufficient to meet the Nyquist criteria. The $100 \mathrm{~mm}$ by $100 \mathrm{~mm}$ imaging field is mapped with a 128 by 128 mesh. In our simulations, the 
thermoacoustic signals are generated in an acoustically inhomogeneous model, while the reconstruction is implemented for two cases--with and without the consideration of acoustic heterogeneity.

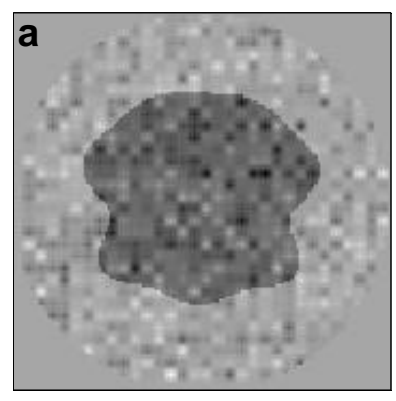

$20 \mathrm{~mm}$

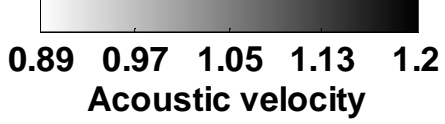

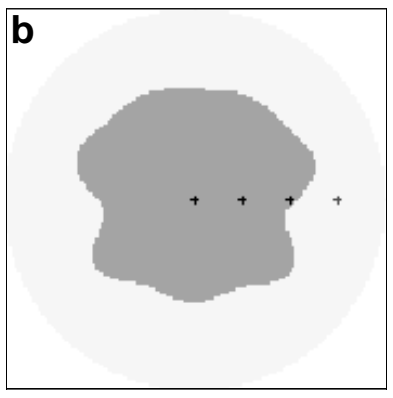

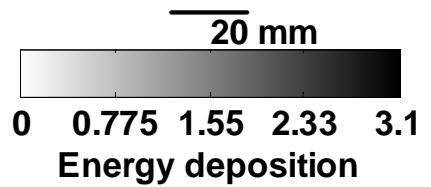

Fig. 3(a) Distribution of acoustic velocity normalized to $v_{s 0}$ for a breast model. The breast surface is represented by the outer circle; the wall between the breast parenchyma and the subcutaneous fat is represented by the inner irregular boundary. (b) The microwave absorption distribution in our model. The four small spots represent the assumed tumors.

\section{NUMERICAL RESULTS}

We first study the effect of acoustic heterogeneity on imaging when acoustic heterogeneity is considered in the forward problem but not in the reconstruction. In the reconstruction, $v_{s}(\mathbf{r})$ in Eq. (9) is set to be $v_{s 0}$. We then show how to improve image resolution after considering acoustic heterogeneity in the reconstructions. Lastly, the effects of measurement errors in $v_{f}, v_{p}$ and $\Sigma$ on the improvement are investigated.
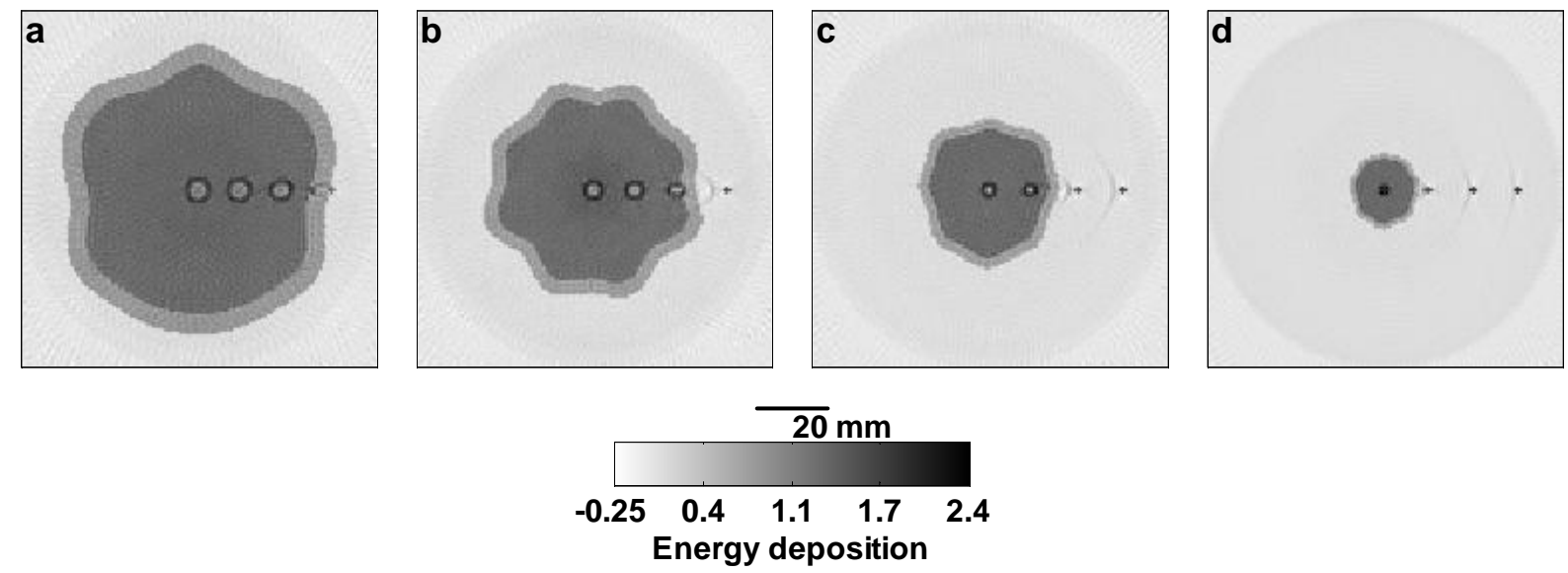

Fig. 4 (a)-(d) Images when acoustic heterogeneity is not considered in the reconstructions. The mean radii of the parenchyma wall are set to be $0.8,0.6,0.4$ and 0.2 of the breast radius, respectively. The point-spread width and the boundary-spread width increase linearly with the size of the parenchyma tissue. Note that the spread of points outside the parenchyma tissue is much smaller than the spread inside. 


\subsection{Reconstruction without considering heterogeneity}

Fig. 4(a)-(d) shows the results when acoustic heterogeneity is not considered in the reconstructions. In the four simulations, the mean radii of the parenchyma wall $r_{p}$ are set to be $0.8,0.6,0.4$ and 0.2 of the breast radius. The wall is distorted randomly in the simulations, and the distortion amplitude is 0.1. We measure the point-spread width (PSW), which is the width of the image of a point source along a specific direction minus its real size, $2.4 \mathrm{~mm}$, and the boundary spread width (BSW), which is the width of the blurred parenchyma wall $\Sigma$ in an image. It is clear from Fig. 4 that PSW and BSW increase with the radius of the parenchyma wall. It is found that the two widths can be estimated by the following equation:

$$
w=l_{p} \alpha,
$$

where $l_{p}$ is $2 r_{p}$ in the case of BSW; in the case of PSW, $l_{p}$ is the length of a ray within the parenchyma tissue along a specific direction. The PSW is anisotropic because $l_{p}$ depends on direction. This anisotropy of PSW can be verified by the observation that the three tumors within the parenchyma tissue in Fig. 4(a) and (b) have the same spread along the horizontal direction, while their spreads along the vertical direction decrease when the tumors are located away from the center.

Another interesting point in Fig. 4 is that the PSW of the objects outside the parenchyma tissue are little affected by acoustic heterogeneity. Only minor artifacts are observed near them. This is because in TAT, a $\pi$ - or wider view can provide complete data for reconstruction ${ }^{24}$. In this case, a view means the angle subtended by the detection curve when observed from the to-be-imaged object. If an object is outside the parenchyma tissue, it has at least a $\pi$ view detection range in which the medium between the object and the detectors is acoustically homogeneous. Therefore, a perfect image can be reconstructed from this part of the data. On the other hand, the image reconstructed from the part of the signals that experience the heterogeneous medium is weak in amplitude because the flight-time errors compromise the build-up strength of the signals.

In addition to the blurring of the images, acoustic heterogeneity increases the background noise level and decreases the values of the reconstructed tumors, which consequently reduces the contrast of the tumors in the images and the detectability of small tumors. A comprehensive quantitative study of this issue will depend on the SNR of the hardware of the imaging system, the parameters of the imaging system and the reconstruction algorithms, and the contrast of the to-be-imaged objects. Meaningful conclusions should be made based on relevant experimental data which we leave for a future study.

\subsection{Reconstruction with the consideration of heterogeneity}

Fig. 5 and Fig. 6 show the reconstructed images with consideration of heterogeneity and the corresponding close-up images around the central tumor in Fig. 5. The exact distribution of acoustic velocity is included in the model in Fig. $\mathbf{5}$ (a). Although the result is good, it is not practical, because it is not feasible to obtain the exact distribution of velocity in the breast with current technology. A much more practical situation is when the mean velocities, $v_{f}, v_{p}$, and boundary profile $\Sigma$ are approximately known while the velocity fluctuation within each area is unknown. Here, we will show the effectiveness of our compensation method. Fig. 5 (b)-(f) shows the images reconstructed from the same data as in Fig. 5 (a), but the reconstruction algorithm used only $v_{f}, v_{p}$ and $\Sigma$ to study the effects of the measurement errors in $v_{f}, v_{p}$ and $\Sigma$ on the improvement. In Fig. 5 (b)-(f), the random component of the acoustic-velocity distribution is ignored. In addition, $v_{p}$ is decreased by $1 \%$ and $3 \%$ in Fig. 5 (c) and (d), respectively; $\Sigma$ is scaled down by $10 \%$ in Fig. 5 (e), and a $20 \%$ random error is introduced to $\Sigma$ in Fig. 5 (f). Fig. 6(a)-(f) are the corresponding close-up images around the central tumor in Fig. 5. $r_{p}$ in these simulations is 0.6 of the breast radius, and the distortion amplitude of the parenchyma wall is 0.2 . 

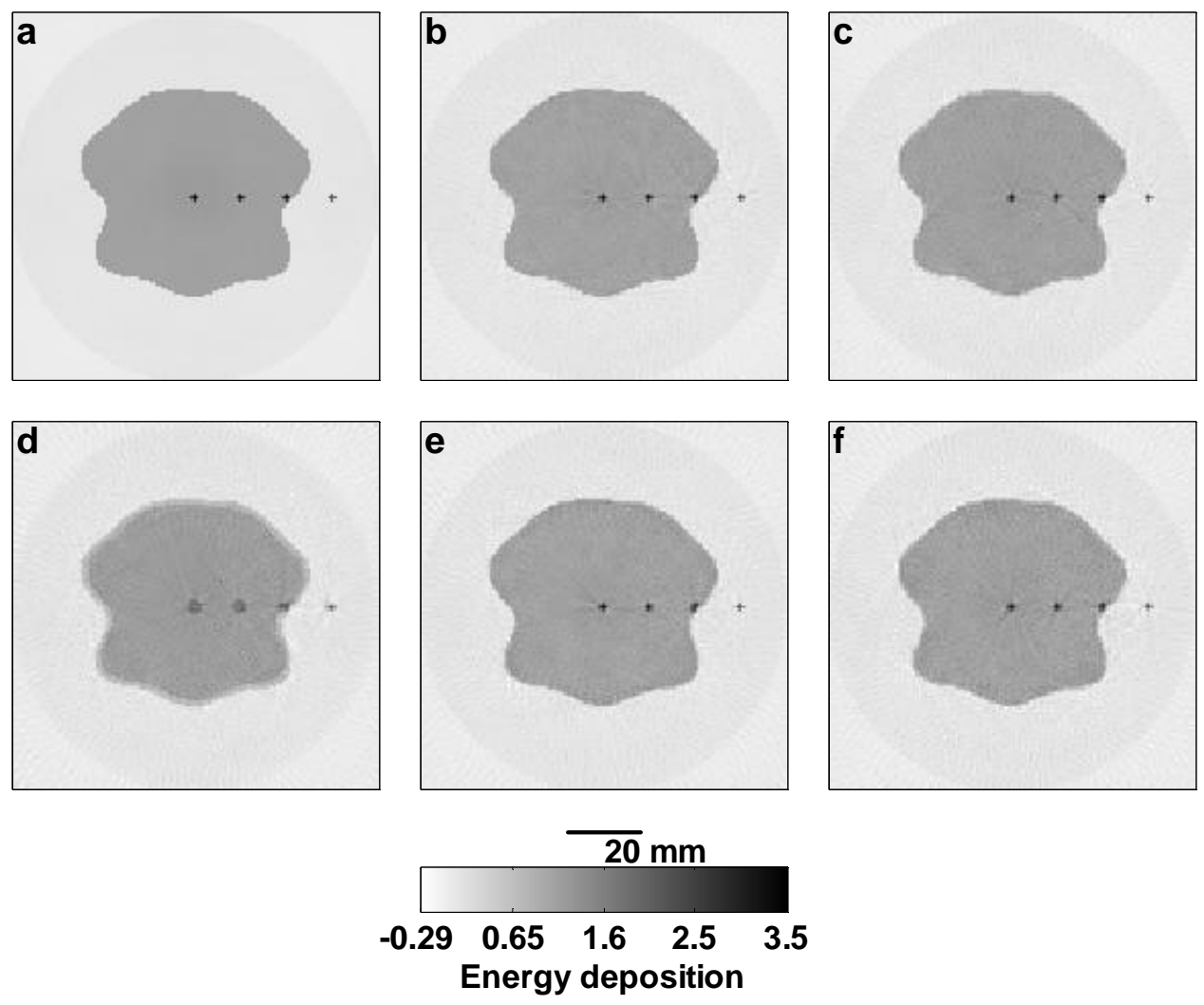

Fig. 5. (a) Compensation for the degradation in images when complete acoustic heterogeneity information is included in the reconstructions. (b) Only exact $v_{p}, v_{f}$, and $\Sigma$ are included to show the insensitivity of improvement to a random component of the acoustic-velocity distribution. (c) and (d) Images when there are $1 \%$ and $3 \%$ errors in $v_{p}$, respectively. (e) Images when $\Sigma$ is scaled down by $10 \%$. (f) Images when $20 \%$ random error is introduced in $\Sigma$. The above results show the stability of the improvement to the errors in $v_{p}, v_{f}$, and $\Sigma$.

\subsubsection{Effect of errors in velocities}

There is little difference between the resolution of the reconstructed images when we consider [Fig. 6 (a)] and do not consider [Fig. 6 (b)], the random component of velocity distribution, although the artifacts in the background in Fig. 6 (b) are a little stronger than those in Fig. 6 (a).

Comparing Fig. 6 (c)-(d) with Fig. 6 (b), it should be noted that a $1 \%$ error in $v_{p}$ does not degrade the imaging quality much, while a $3 \%$ error in $v_{p}$ greatly deteriorates the imaging resolution and contrast. This is because in our model, the difference between $v_{f}$ and $v_{p}$ is about $7 \%$ of their speeds, and a $3 \%$ error in $v_{p}$ actually accounts for $42 \%$ of the difference between $v_{f}$ and $v_{p}$. Therefore, we conclude that an accuracy of $1 \%$ in the determination of $v_{p}$ is sufficient for significant improvement in imaging resolution. 

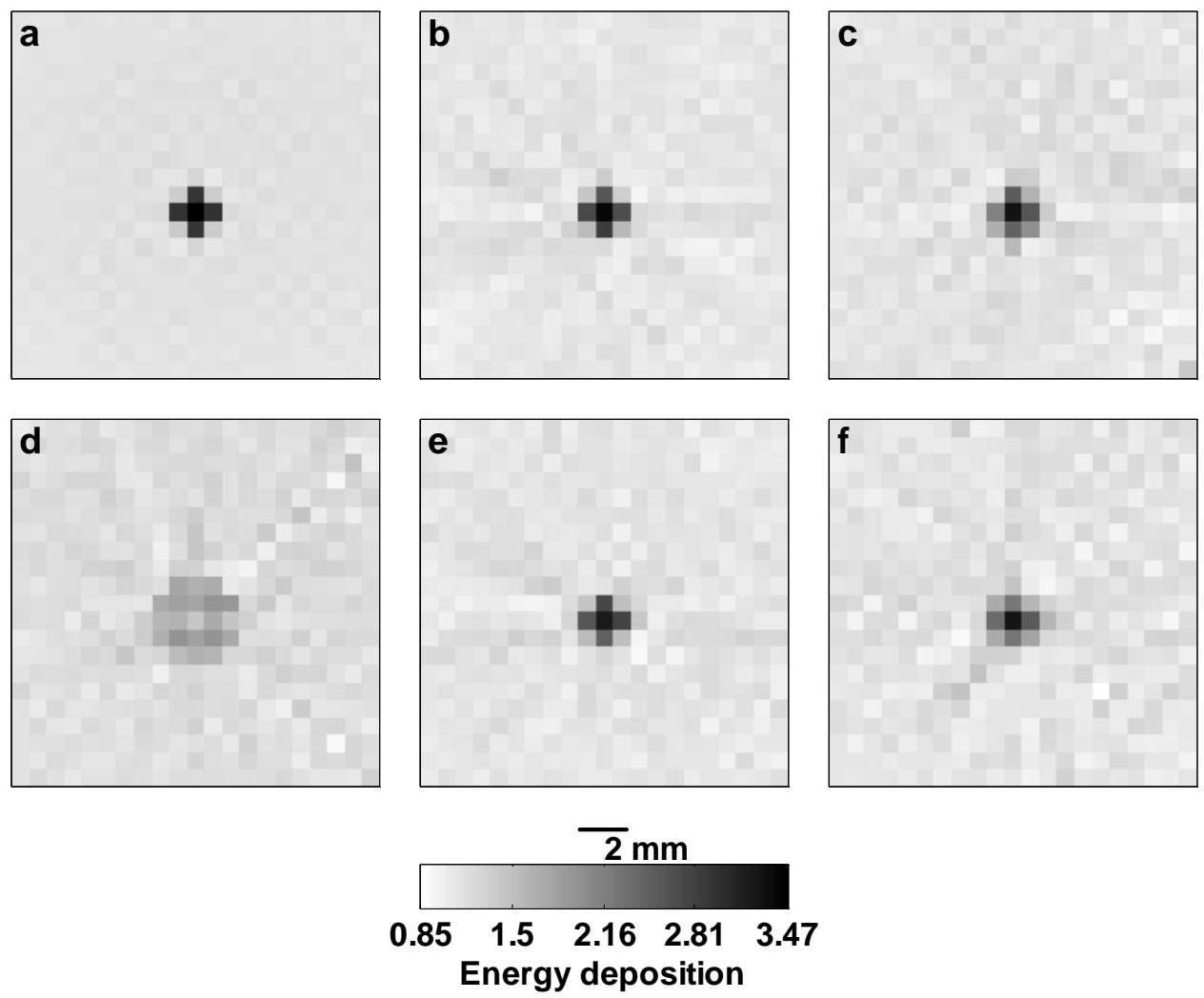

Fig. 6(a)-(f) Close-up images around the central tumor in Fig. 5 (a)-(f), respectively.

\subsubsection{Effects of errors in determining $\Sigma$}

In the model in Fig. 6 (e), the boundary $\Sigma$ is scaled down by 10\%. In Fig. 6 (f), a random component is added to the real boundary, which is implemented by multiplying the real radii of a boundary with uniform random numbers within [0.8,1.2]. After comparing Fig. 6 (e) and (f) with other figures in Fig. 6, it is found that compensation is less sensitive to error in determining $\Sigma$ as $v_{p}$. This is because a $10 \%$ error, which is about $6 \mathrm{~mm}$ in the diameter of the parenchyma wall, adds at most $0.42 \mathrm{~mm}$ to the PSW and BSW according to Eq. (10).

\section{DISCUSSION}

The studies we presented in Section 3 show that there should be no severe amplitude distortion in breast TAT although severe amplitude distortion caused by refraction has been observed in both narrowband and broadband breast $\mathrm{UT}^{11}$. The difference between the effects of acoustic heterogeneity on TAT and UT can be explained by the different central frequencies. In UT, the central frequency is above $3 \mathrm{MHz}$, while in TAT, the central frequency is below $1 \mathrm{MHz}$. The higher frequency in UT results in stronger wavefront distortion for the following reasons. First, the scattering effect increases rapidly with frequency; and secondly, the minimum detection distance for avoiding strong amplitude distortion caused by an acoustic lens, which can be a boundary segment or a small inclusion, extends farther with increasing frequency. We notice that the transducer or array was placed closer than the required distance to the breast ${ }^{11,12}$. Therefore, it is not surprising to observe the strong interference effect in UT.

Another important difference between TAT and UT is that there is no speckle in our TAT images ${ }^{7}$. Speckle is an important factor limiting the quality of pure ultrasonic imaging. In our technology, the detected signals are primary acoustic waves, rather than reflective or scattered waves as in UT. Further, the temporal frequency of the acoustic signals lies in a range from 0 to $1.5 \mathrm{MHz}$, which is only weakly scattered in the tissues. However, the issue of image speckle in more realistic medical imaging applications is a topic for future consideration. 


\section{CONCLUSIONS}

The effects of acoustic heterogeneity on TAT in the breast are studied. Our analysis shows that the amplitude distortion in breast TAT is minor. The amplitude distortion is not severe in breast TAT, because the TAT signals are broadband, have low central frequency, and experience only one-way transmission through the parenchyma wall. Therefore, we consider only phase distortion in our numerical studies. The numerical results on the spread of point sources and boundaries caused by the phase distortion are in good agreement with the predictions of the proposed formula. It is shown that phase distortion can be compensated for when complete or partial information on the distribution of acoustic velocity in the breast is included in the reconstruction. It is discovered that improvement in the results is more sensitive to measurement error in $v_{f}, v_{p}$ than in $\Sigma$. The differences between breast TAT and breast ultrasound imaging in relation to the effects of acoustic heterogeneity and speckles are accounted for by differences between them in their central ultrasound frequencies and detection configurations.

\section{ACKNOWLEDGMENTS}

We would like to thank Dr. Q. Zhu for many useful discussions. This project was sponsored in part by the U.S. Army Medical Research and Materiel Command Grant No. DAMD17-00-1-0455, the National Institutes of Health Grants No. R01 CA71980 and No. R21 CA83760, the National Science Foudation Grant No. BES-9734491, and Texas Higher Education Coordinating Board Grant No. ARP 000512-0063-2001.

\section{REFERENCES}

1 W. Joines, R. Jirtle, M. Rafal, and D. Schaeffer, "Microwave power absorption differences between normal and malignant tissue," Radiation Oncology-Biology-Physics vol. 6, pp. 681-687, 1980.

2 S. Chaudhary, R. Mishra, A. Swarup, and J. Thomas, "Dielectric properties of normal human the breast tissues at radiowave and microwave frequencies," Indian Journal of Biochemistry and Biophysics vol. 21, pp. 76-79, 1984.

3 W. Joines, Y. Zhang, C. Li, and R. Jirtle, "The measured electrical properties of normal and malignant human tissues from 50-900 MHz," Medical Physics vol. 21, pp. 547-550, 1994.

4 R. A. Kruger, P. Liu, Y. R. Fang, and C. R. Appledorn, "Photoacoustic ultrasound (PAUS)—reconstruction tomography," Med. Phys. vol. 22, pp. 1605-1609, 1995.

5 C. G. A. Hoelen, F. F. M. Demul, R. Pongers, and A. Dekker, "Three-dimensional photoacoustic imaging of blood vessels in tissue," Opt. Lett. vol. 23, pp. 648-650, 1998.

6 G. Ku and L.-H. V. Wang, "Scanning thermoacoustic tomography in biological tissue," Med. Phys. vol. 27, pp. 1195-1202, 2000.

7 Y. Xu, D. Feng, L.-H. V. Wang, "Exact frequency-domain reconstruction for thermoacoustic tomography: I. Planar geometry," IEEE Trans. Med. Imag., vol. 21, pp. 823 - 828, 2002.

$8 \quad$ M. Xu and L.-H. V. Wang, "Time-domain reconstruction for thermoacoustic tomography in a spherical geometry," IEEE Trans. Med. Imag., vol. 21, pp. $814-822,2002$.

9 Y. Xu, M. Xu, L. -H V. Wang, "Exact frequency-domain reconstruction for thermoacoustic tomography: II. Cylindrical geometry," IEEE Trans. Med. Imag., vol. 21, pp. 829 - 833, 2002.

10 M. Moshfeghi and R. C. Waag, "In vivo and in vitro ultrasound beam distortion measurements of a large aperture and a conventional aperture focused transducer," Ultrsound Med. Bio., vol. 5, pp. 415-428, 1988.

11 Q. Zhu and B. D. Steinberg, "Large-transducer measurements of wavefront distortion in female the breast," Ultrasonic imag. Vol. 14, pp. 276-299, 1992.

12 C. W. Manry and S. L. Broschat, "FDTD simulations for ultrasound propagation in a 2-D breast model," Ultra. Imag. Vol. 18, pp. 25-34, 1996.

13 P. D. Freiburger, D. C. Sullivan, B. H. Leblanc, S. W. Smith, and G. E. Trahey, "Two dimensional ultrasonic beam distortion in the breast: in vivo measurements and effects," Ultra. Imag. Vol. 14, pp. 398-414, 1992.

14 S. W. Flax and M. O'Donnell, "Phase aberration correction using signals from point reflectors and diffuse scatters: basic principles,” IEEE Trans. Ultrason. Ferroelec. Freq. Contr., vol. 35, pp. 758-767, 1988. 
G. E. Trahey, D. Zhao, J. A. Miglin and S. W. Smith, "Experimental results with a real-time adaptive ultrasonic imagining system for viewing through distorting media, IEEE Trans. Ultrason. Ferroelec. Freq. Contr., vol. 37, pp. 418-427, 1990.

16 Q. Zhu and B. D. Steinberg, "Deaberration of incoherent wavefront distortion: an approach toward inverse filtering,” IEEE Trans. Ultrason. Ferroelec. Freq. Contr., vol. 44, pp. 575-589, 1997.

17 Y. Xu and L.-H. V. Wang, "Signal processing in scanning thermoacoustic tomography in biological tissues," Medical Physics, vol. 28, pp. 1519-1524, 2001.

18 Hansen P. C., "Rank-Deficient and Discrete Ill-Posed Problems", Philadelphia, PA: SIAM Press, 1998.

19 Y. Xu and L.-H. V. Wang, "Effects of acoustic heterogeneity in breast thermoacoustic tomography," IEEE Trans. Ultrason. Ferroelec. Freq. Contr., in print, 2003.

20 A. R. Selfridge, "Approxiamte properties in isotropic materials," IEEE Trans. On Sonics and Ultra., SU-32, pp. 381-394, 1985.

21 R. Sneider, D. F. Aldridge, "Perturbation theory for travel times," J. Acoust. Soc. Am., vol. 98, pp. 1565-1569, 1995.

22 J. F. Greenleaf, R. C. Bahn, "Clinical imaging with transmissive ultrasonic computerized tomography", IEEE Trans. Biom, Engineering, Vol. BME-28, N0. 2, 177-185, 1981.

23 F. S. Foster, M. Strban, and G. Austin, "The ultrasound microscope: initial studies of the breast tissue," Ultra. Imag. Vol. 6, pp. 243-261, 1984.

24 Y. Xu and L.-H. V. Wang, "Limited-view thermoacoustic tomography and reconstruction by truncated-conjugate gradient," IEEE Trans. Med. Imag., in revision, 2002. 